\title{
Personality and externalizing behavior in the transition to young adulthood: the additive value of personality facets
}

\author{
Theo A. Klimstra $\cdot$ Koen Luyckx • \\ William W. Hale III $\cdot$ Luc Goossens
}

Received: 4 April 2013/Accepted: 17 January 2014/Published online: 31 January 2014

(C) Springer-Verlag Berlin Heidelberg 2014

\begin{abstract}
Purpose The directionality of effects in the associations between personality and externalizing behavior (i.e., delinquency, soft drugs use, and alcohol abuse) is unclear. Moreover, previous studies only examined personality trait domains when examining these associations, whereas personality facets underlying these broad domains provide more specificity. To address these limitations, the present study examined the directionality of effects between externalizing behavior and personality while employing a facet-level approach to personality.

Methods Cross-lagged panel models were employed to four annual measurement waves of longitudinal data on 485 Belgian-Caucasian late adolescents $(87.4 \%$ female; $M_{\text {age }}=18.63$ years, $\left.\mathrm{SD}=0.61\right)$. Participants filled out the NEO-FFI as a measure of personality, the Deviant Behavior Scale as a measure of delinquency, and single items for soft drugs use and alcohol abuse on all four measurement occasions.

Results The incremental value of personality facets over broad trait domains was demonstrated, as it was often the case that only some, but not all, facets underlying a specific trait domain were significantly associated with specific
\end{abstract}

T. A. Klimstra (凹)

Department of Developmental Psychology, Tilburg University, Postbus 90153, 5000 LE Tilburg, The Netherlands

e-mail: t.a.klimstra@uvt.nl; theoklimstra@online.nl

K. Luyckx · L. Goossens

School Psychology and Child and Adolescent Development, KULeuven, University of Leuven, Leuven, Belgium

W. W. Hale III

Research Centre Adolescent Development, Utrecht University, Utrecht, The Netherlands externalizing behaviors. Furthermore, linkages between personality and externalizing behavior were shown to be complex. Depending on the personality trait domain or facet and the specific behavior under investigation, the directionality of effects may differ.

Conclusions To capture the full complexity of the linkages between personality and externalizing behavior, a facet approach to personality is recommended. This information is potentially important for clinicians, as it indicates which specific aspects of a broad trait domain affect, and are affected by, specific externalizing behaviors.

Keywords Personality $\cdot$ Big five $\cdot$ Externalizing problems $\cdot$ Adolescence $\cdot$ Personality facets

\section{Introduction}

Late adolescents and young adults are relatively independent from their parents, yet they have few enduring adultlike responsibilities [1]. They are expected to explore several alternatives with regard to relationships, their professional career, and ideology to find their own place in society. During the course of this exploration process, they may experiment with (soft) drugs and alcohol or engage in minor delinquent acts [2].

Occasional soft drugs use, alcohol abuse, and even minor delinquency are common [2] and may, therefore, be considered normative in this period in life, but in some individuals these behaviors exacerbate. Therefore, uncovering predictors of individual differences in externalizing behaviors is important. Several studies suggest that personality traits are associated with soft drugs use [e.g., 3], alcohol abuse [e.g., 4], and (minor) delinquency [e.g., 5]. However, these studies tend to have a number of 
limitations. First, few studies include multiple longitudinal measurement occasions of both personality traits and externalizing behaviors. Such a design is necessary to infer directionality of effects. Second, broad constructs of externalizing behavior are often employed. Such broad constructs ignore, for example, that soft drugs use is different from minor delinquency. Third, previous studies only considered personality trait domains. Such trait domains entail a wide range of more specific facets. The purpose of the present study is to provide a detailed perspective on the linkages between personality and externalizing behavior by (a) employing a longitudinal design, (b) distinguishing between three externalizing behaviors (i.e., soft drugs use, alcohol abuse, and minor delinquency), and (c) adopting a facet-level approach to personality.

\section{Personality traits and externalizing behavior}

Personality at the trait-domain level is usually captured by the Big Five [6, 7]. These trait domains are Neuroticism (i.e., tendencies toward negative affect), Extraversion (i.e., experiencing frequent positive moods and sociable tendencies), Openness (i.e., tendencies toward curiosity and creativity), Agreeableness (i.e., tendencies toward prosocial behavior), and Conscientiousness (i.e., tendencies towards being planful, responsible, and persevering) [8]. These traits have been frequently linked to problem behavior.

Several models have been proposed to describe the relations between personality and problem or externalizing behavior $[9,10]$. The first set of models, the predisposition or vulnerability model and the pathoplasty or exacerbation model, state that personality traits predict changes in externalizing behavior. However, whereas the vulnerability model states that individuals with specific personality characteristics (e.g., low agreeableness) but without current psychopathology are more vulnerable to develop psychopathology (e.g., Conduct Disorder) later on [11], the pathoplasty model states that specific personality traits predict individual differences in the course, severity, presentation, and prognosis of externalizing behavior. Because the pathoplasty model also includes personality effects in explaining variation in the severity of externalizing behavior, this model better describes effects of personality traits on externalizing behavior in the general population. Findings of previous studies on longitudinal effects of personality on problem behavior are therefore best described as pathoplasty rather than vulnerability effects.

Second, the complication or scar model states that high levels of problem behavior may damage one's personality (e.g., predict low conscientiousness). Thus, the assumption of such a model is that externalizing behavior would lead to changes in personality traits $[9,10]$. It should be noted that the aforementioned models are not mutually exclusive, as a study on the longitudinal linkages between personality, depression, and aggression [12] found evidence for effects of personality on problem behavior and of problem behavior on personality occurring simultaneously.

Finally, the spectrum model assumes that personality traits and problem behavior are situated on the same continuum. For example, minor delinquency could be a manifestation of low agreeableness. If so, increases in agreeableness should be correlated with decreases in minor delinquency. Therefore, to test the spectrum model, one should examine whether changes in personality traits and problem behavior are correlated [13]. Evidence for these different models will now be discussed for (minor) delinquency, soft drugs use, and alcohol abuse.

\section{Delinquency}

Associations between Big Five personality traits and delinquency are well documented. A meta-analysis [5] revealed strong negative associations of delinquency with conscientiousness and agreeableness, and a much weaker positive association of delinquency with neuroticism.

Most longitudinal studies examined pathoplasty models. Findings of one of these studies [14] were identical to the results of the meta-analysis by Miller and Lynam [5]: low conscientiousness and agreeableness, and high neuroticism predicted delinquency across adolescence. Shiner [15] obtained similar results, with early adolescent agreeableness and academic conscientiousness positively predicting late adolescent rule abiding conduct. In a study on children [16], a similar effect of agreeableness was obtained, but an additional effect of low extraversion was also found. Overall, especially agreeableness and conscientiousness, and to a lesser extent neuroticism, seem to be the best predictors of delinquency.

We found only one study examining a scar model for delinquency [17]. In that study, delinquency predicted constraint (i.e., related to conscientiousness) negatively, and negative emotionality (i.e., related to neuroticism) positively across adolescence. To the best of our knowledge, studies examining pathoplasty and scar models simultaneously, and longitudinal studies examining the spectrum model are non-existent.

\section{Soft drugs use}

Several studies linked personality traits to soft drugs use (mainly marihuana use). Marihuana use was found to be negatively associated with conscientiousness in a metaanalysis specifically focused on this trait [18]. Subsequent cross-sectional studies [3, 19] also found positive 
associations with openness and negative associations with agreeableness.

A longitudinal study [20] provided some support for a pathoplasty model, as marihuana use was positively predicted by neuroticism and negatively predicted by conscientiousness. In another study [21], childhood hostility, a trait related to neuroticism, positively predicted marihuana use through mid-adolescence.

One study [22] examined reciprocal linkages of marihuana use with traits related to agreeableness and conscientiousness (i.e., responsibility, temperance, and perspective-taking). This study demonstrated that marihuana use predicted smaller increases in these traits, which, in turn, predicted smaller increases in marihuana use. Thus, these findings suggest simultaneous scar and pathoplasty effects. However, longitudinal studies simultaneously examining scar and pathoplasty models while specifically focusing on Big Five personality traits appear to be nonexistent.

\section{Alcohol abuse}

Linkages between personality and problematic alcohol use have been examined in many studies. Three meta-analyses collectively suggest that alcohol abuse is positively associated with neuroticism, and negatively associated with conscientiousness and agreeableness [4, 18, 23]. There is also evidence for correlated change, as decreases in problematic alcohol involvement were found to be associated with increases in conscientiousness and decreases in neuroticism [24]. Hence, problematic alcohol use seems to lay on the same continuum as (i.e., is a behavioral manifestation of) low conscientiousness and high neuroticism, supporting a spectrum model.

Several studies support a pathoplasty model for the linkages between alcohol abuse and personality traits. Especially high levels of neuroticism, extraversion, and related traits (i.e., hostility and sociability, respectively) seem to leave individuals vulnerable to alcohol abuse [20, 21].

Few studies have examined scar models for the linkages between personality and alcohol abuse. The aforementioned study by Chassin and colleagues [22] also examined transactional linkages between alcohol use and traits related to agreeableness and conscientiousness. In line with the scar model, they showed that alcohol use predicted smaller increases in these traits, but that these traits did not predict changes in alcohol use. Hicks et al. [25] showed that decreases in behavioral disinhibition (being inversely related to conscientiousness) were greater for adolescents whose alcohol abuse desisted when compared to adolescents who persisted with alcohol abuse into their twenties. This finding suggests that alcohol abuse positively predicts behavioral disinhibition, supporting a scar model. However, behavioral disinhibition and negative emotionality (being comparable to neuroticism) also predicted alcohol abuse. Thus, their findings suggested simultaneously occurring pathoplasty and scar effects. We are unaware of studies assessing reciprocal linkages between Big Five traits and alcohol use.

Overall, studies addressing the linkages between personality traits and externalizing behaviors all share one important limitation: They tend to consider only broad trait domains. Such a trait-domain level approach only yields a 'quick-and-dirty' perspective on personality [26]. To gain a more detailed perspective on personality and its associations with other constructs such as externalizing behavior, specific facets underlying broad trait domains need to be considered.

\section{The additive value of personality facets}

Personality is hierarchically structured with specific facets underlying broad trait domains. For example, in the trait domain of neuroticism, facets of anxiety and depression can be distinguished, whereas conscientiousness can be subdivided into orderliness, goal-directedness, and selfdiscipline [e.g., 26-28]. As these facets tap into different aspects of the same overarching trait domain, they can be differently associated with externalizing behaviors.

For delinquency, studies on associations with Big Five facets are non-existent. However, associations with facets of a three-dimension personality model [29] have been examined [30]. Some facets underlying a specific trait domain were associated with delinquency, whereas others were not. For example, the aggression facet of the negative emotionality domain positively predicted antisocial behavior, whereas the stress reaction facet of this domain was no significant predictor in this regard. Ge and Conger [17] considered facets of the same model to test a scar model. The utility of facets was again underscored, as delinquency significantly predicted some facets underlying specific trait domains, whereas other facets underlying the same trait domain were not significantly predicted by delinquency.

A meta-analysis of cross-sectional studies on soft drugs (i.e., marihuana) use focused on negative affect and emotionality (i.e., facets related to neuroticism) and unconventionality (i.e., a facet of openness) [31]. This study found little evidence for differential associations, as all these facets were positively associated with marihuana use. Terraciano and colleagues [3] did find evidence for the added value of facets in an examination of a pathoplasty model while considering six facets for each trait domain. Marihuana use was positively predicted by the 
openness facets values and ideas, the neuroticism facets angry hostility and vulnerability, and the extraversion facets activity and excitement seeking. Other facets of these trait domains did not predict marihuana use. Likewise, only the conscientiousness facets dutifulness and deliberation, and the agreeableness facet compliance negatively predicted marihuana use.

With regard to alcohol use, one study considered 6 facets per Big Five trait domain [32]. Cross-sectionally, alcohol use was consistently positively associated with the extraversion facets of gregariousness, friendliness, and excitement seeking, and the neuroticism facet of immoderation. agreeableness facets of morality and cooperation and conscientiousness facets of dutifulness, achievement striving, and cautiousness were consistently negatively associated with alcohol use. Other facets underlying these traits were unassociated with alcohol use. A pathoplasty model was also examined. Only extraversion and its facets gregariousness and cheerfulness (but not the other four facets of extraversion) positively predicted relative increases in alcohol use. Overall, there was again clear evidence for the additive value of facets over trait domains.

In sum, there have been some studies linking externalizing problems to personality facets. However, many of these studies were cross-sectional, whereas the few available longitudinal studies either tested a pathoplasty model or a scar model, but never tested both models simultaneously. A reason for this may be that testing pathoplasty and scar models simultaneously requires multiple measurement occasions of both externalizing problems and personality facets. Including multiple measurement occasions of personality facets may be thought of as impracti$\mathrm{cal}$, as measuring facets is often thought to require lengthy questionnaires such as the NEO-PI-R and the NEO-PI-3 (240 items) [33]. However, it is also possible to use much shorter questionnaires to measure personality at a facet level. That is, facet scales have been recently developed for the 44-item Big Five Inventory [28]. Furthermore, facet scales are also available for the commonly used 60-item NEO-FFI [34].

Using exploratory factor analysis, Saucier [27] identified a total of 14 facets ( 2 or 3 per Big Five domain) in the NEO-FFI. Specifically, neuroticism contained a selfreproach, depression, and anxiety facet; extraversion an activity, sociability, and positive affect facet; openness an esthetic interests, intellectual interests, and unconventionality facet; agreeableness a pro-social orientation and nonantagonistic orientation facet; and conscientiousness an orderliness, goal striving, and dependability facet. These facets had sufficient divergent validity, as evidenced by differential correlations with the 30 facets of the NEO-PI-R and person-descriptive adjectives.
Chapman [35] replicated the same 14 facets with confirmatory factor analyses. Divergent validity of the NEOFFI facets was further demonstrated, as facets underlying the same trait were differently associated with external variables. For example, the extraversion facet of sociability was a better predictor of loneliness than the extraversion facet of activity. Thus, using the NEO-FFI to assess personality at a facet level represents a viable alternative to using lengthier questionnaires. This opportunity may be particularly appealing because many researchers may have longitudinal NEO-FFI data without being aware of the existence of these facet scales.

\section{The present study}

In the present study, we used personality facets to gain a detailed perspective on the longitudinal associations of personality with externalizing behaviors. Four annual waves of longitudinal data on personality (i.e., domains and facets) and three externalizing behaviors (i.e., minor delinquency, soft drugs use, and alcohol abuse) were employed to examine whether a pathoplasty model, a scar model, both a scar and a pathoplasty model, or a spectrum model best described these linkages. It should be noted that we used cross-lagged models focusing on the prediction of relative changes in a variable [36]. That is, a significant (cross-) path from, for example, orderliness to delinquency, in such a model would indicate that the rank-order of individuals on orderliness on one measurement occasion predicts changes in the rank-order of individuals on delinquency on the subsequent measurement occasion. Thus, a negative path from orderliness to delinquency would indicate that individuals with a relatively lower score on orderliness would be more likely to exhibit relative increases (i.e., a move up in the rank-order) of delinquency on the next measurement occasion. Therefore, we do not claim that we used personality scores to predict whether a person exhibited absolute increases in externalizing behavior scores when we refer to the pathoplasty model. Likewise, we do not claim that we used externalizing behavior scores to predict absolute increases in scores on neuroticism and its facets or decreases in scores on the other Big Five trait domains and their facets when we refer to the scar model.

To the best of our knowledge, there are no studies available that test all these models for the linkages of personality traits and facets with minor delinquency, soft drugs use, and alcohol abuse. There is, however, one study that tests these models for the linkages between Big Five personality trait domains and a broad externalizing behavior construct [13]. The results of that study were mostly in line with the spectrum model. However, it is 
unclear whether similar results will be obtained if specific externalizing behaviors are considered and if personality facets are considered in addition to trait domains.

We can provide specific hypotheses with regard to which personality traits and facets are expected to be associated with the three externalizing behaviors examined. Minor delinquency is expected to be associated with low agreeableness and conscientiousness. Given a lack of previous facet-level Big Five studies, we have no specific hypotheses with regard to facets. Soft drugs use is expected to be positively associated with openness, and negatively associated with agreeableness and conscientiousness. At the facet level, the openness facet unconventionality and the extraversion facet activity are expected to be positively associated with soft drugs use, whereas the agreeableness facet non-antagonistic orientation and the conscientiousness facet dependability are expected to be negatively associated. We expect alcohol abuse to be associated with low agreeableness and conscientiousness, and high neuroticism and extraversion. At the facet level, the extraversion facet of sociability is expected to be positively associated with alcohol use, whereas the conscientiousness facets of goal striving and dependability and the agreeableness facet of non-antagonistic orientation are expected to be negatively associated.

\section{Method}

Participants and procedure

Participants were drawn from the Leuven Trajectories of Identity Development Study (L-TIDES) [37], a 7-wave longitudinal study of Dutch-speaking college students from the faculty of Psychology and Educational Sciences at a large Belgian university. Permission to undertake this study was granted by the Institutional Review Board within the researchers' department and have therefore been performed in accordance with the ethical standards laid down in the 1964 Declaration of Helsinki and its later amendments. Participants signed a standard informed consent form before participating in the first wave of data collection. During the informed consent process, participants were informed that they could refuse or discontinue participation at any time.

The first wave was collected at the end of 2002, and data on both personality and externalizing behavior were available for four measurement occasions. These four waves (referred to as T1, T2, T3, and T4 from this point onwards) were used in the present study. There was a 1-year interval between each of these four waves. At Time 1 , a total of 565 individuals participated. Only individuals who participated in at least two out of four measurement waves $\left(N=485 ; 87.4 \%\right.$ female; $M_{\text {age }}=18.63$ years,
$\mathrm{SD}=0.61)$ were included in the analyses. MANOVAs indicated that the 485 included participants did not differ significantly from the 80 excluded participants in terms of personality $(F(19,545)=1.41 ; p=0.118)$ or externalizing behavior $(F(3,561)=2.47 ; p=0.061)$ at Time 1 (i.e., the only measurement occasion on which we had complete data for both groups). Overall, $14.71 \%$ of the data were missing for the 485 included participants. Participants with and without complete data were compared using Little's [38] Missing Completely At Random (MCAR) test. This test yielded a $\chi^{2} / d f$-value of 1.13 , which according to guidelines by Bollen [39] indicates a good fit between sample scores with and without imputation. Therefore, we used the Full Information Maximum Likelihood procedure in subsequent analyses.

Measures

\section{Personality traits}

Personality was measured with the Dutch version of the 60-item NEO Five-Factor Inventory (NEO-FFI) [34, 40]. This instrument was originally developed to assess Big Five domains (i.e., neuroticism, extraversion, openness to experience, agreeableness, and conscientiousness), but facets underlying these domains can also be distinguished [27, 35]. For neuroticism, facets of self-reproach, anxiety, and depression can be distinguished. For extraversion, there are facets of sociability, positive affect, and activity. Openness is subdivided in facets of esthetic interests, intellectual interests, and unconventionality. The facets for agreeableness are non-antagonistic orientation and prosocial orientation. Finally, the conscientiousness facets are orderliness, goal striving, and dependability.

Though facets were measured with relatively few items, Cronbach's alphas for all domains and 10 out of 14 facets were consistently above 0.55 across waves (see Table 1). However, the extraversion facet of sociability and the conscientiousness facet of dependability had problematic Cronbach's alphas (i.e., <0.55) on some measurement waves, but were reliable on others. Cronbach's alphas of the openness facet unconventionality and the agreeableness pro-social orientation were consistently below 0.55 (i.e., 0.28 and 0.43 on average, respectively). Therefore, analyses involving these facets should be interpreted cautiously. The scale scores we used in our analyses were derived by calculating the means across the items representing each of the domain scales and facet scales.

\section{Delinquency}

Delinquency was assessed with 11 items adapted from the Deviant Behavior Scale (DBS) [41]. Participants were 
Table 1 Reliability and rank-order stability of personality domains and facets, and adjustment $(N=485)$

\begin{tabular}{|c|c|c|c|c|c|c|c|}
\hline \multirow[t]{2}{*}{ Domain/facet } & \multicolumn{4}{|c|}{ Reliability } & \multicolumn{3}{|c|}{ Rank-order stability } \\
\hline & $\mathrm{T} 1$ & $\mathrm{~T} 2$ & $\mathrm{~T} 3$ & $\mathrm{~T} 4$ & $\mathrm{~T} 1-\mathrm{T} 2$ & $\mathrm{~T} 2-\mathrm{T} 3$ & $\mathrm{~T} 3-\mathrm{T} 4$ \\
\hline Neuroticism & 0.87 & 0.88 & 0.89 & 0.89 & $0.63 * * *$ & $0.68 * * *$ & $0.67 * * *$ \\
\hline $\mathrm{N}$ self-reproach & 0.72 & 0.76 & 0.77 & 0.78 & $0.62 * * *$ & $0.76^{* * *}$ & $0.70 * * *$ \\
\hline $\mathrm{N}$ anxiety & 0.69 & 0.68 & 0.68 & 0.68 & $0.61 * * *$ & $0.68 * * *$ & $0.66 * * *$ \\
\hline $\mathrm{N}$ depression & 0.71 & 0.75 & 0.74 & 0.70 & $0.66 * * *$ & $0.65 * * *$ & $0.62 * * *$ \\
\hline Extraversion & 0.81 & 0.82 & 0.80 & 0.81 & $0.68 * * *$ & $0.67 * * *$ & $0.65 * * *$ \\
\hline E positive affect & 0.81 & 0.84 & 0.83 & 0.83 & $0.73 * * *$ & $0.70 * * *$ & $0.72 * * *$ \\
\hline E sociability & 0.60 & 0.50 & 0.50 & 0.53 & $0.61 * * *$ & $0.63 * * *$ & $0.68 * * *$ \\
\hline E activity & 0.60 & 0.63 & 0.63 & 0.56 & $0.66 * * *$ & $0.66^{* * *}$ & $0.66 * * *$ \\
\hline Openness & 0.71 & 0.72 & 0.75 & 0.73 & $0.68 * * *$ & $0.67 * * *$ & $0.71 * * *$ \\
\hline $\mathrm{O}$ esthetic interests & 0.74 & 0.76 & 0.78 & 0.79 & $0.71 * * *$ & $0.75^{* * *}$ & $0.80 * * *$ \\
\hline O intellectual interests & 0.58 & 0.59 & 0.67 & 0.69 & $0.67 * * *$ & $0.66 * * *$ & $0.69 * * *$ \\
\hline O unconventionality & 0.32 & 0.25 & 0.38 & 0.17 & $0.52 * * *$ & $0.58 * * *$ & $0.60 * * *$ \\
\hline Agreeableness & 0.69 & 0.70 & 0.71 & 0.71 & $0.58 * * *$ & $0.64 * * *$ & $0.63 * * *$ \\
\hline A non-antagonistic orientation & 0.62 & 0.63 & 0.64 & 0.61 & $0.64 * * *$ & $0.67 * * *$ & $0.65 * * *$ \\
\hline A pro-social orientation & 0.37 & 0.46 & 0.42 & 0.46 & $0.47 * * *$ & $0.61 * * *$ & $0.48 * * *$ \\
\hline Conscientiousness & 0.79 & 0.82 & 0.84 & 0.84 & $0.68 * * *$ & $0.71 * * *$ & $0.66 * * *$ \\
\hline $\mathrm{C}$ orderliness & 0.74 & 0.75 & 0.75 & 0.78 & $0.74 * * *$ & $0.77 * * *$ & $0.74 * * *$ \\
\hline C goal striving & 0.61 & 0.58 & 0.66 & 0.64 & $0.62 * * *$ & $0.70 * * *$ & $0.67 * * *$ \\
\hline $\mathrm{C}$ dependability & 0.47 & 0.60 & 0.60 & 0.57 & $0.56 * * *$ & $0.61 * * *$ & $0.68 * * *$ \\
\hline Alcohol abuse & \multicolumn{4}{|c|}{ Single item } & $0.65 * * *$ & $0.73 * * *$ & $0.77 * * *$ \\
\hline Soft drug use & \multicolumn{4}{|c|}{ Single item } & $0.71 * * *$ & $0.76 * * *$ & $0.86 * * *$ \\
\hline Delinquency (excl. SU) & 0.76 & 0.69 & 0.60 & 0.66 & $0.58 * * *$ & $0.69 * * *$ & $0.72 * * *$ \\
\hline
\end{tabular}

Note. Cronbach's alphas cannot be computed for single-item measures $* * * p<0.001$

asked to indicate on a 5-point Likert-type rating scale (ranging from "does not apply to me at all" to "applies to me very well") the degree to which they engaged in deviant behaviors (such as shoplifting and destroying other people's property) during the past 6 months. The scale was reliable, as Cronbach's alphas ranged from 0.60 to 0.76 across waves. The scale scores we used in our analyses were derived by calculating the mean across the 11 items of this scale.

\section{Alcohol abuse}

Alcohol abuse in the last 6 months was measured with a single item adapted from the DBS [41]. This 5-point Likert scale (ranging from "does not apply to me at all" to "applies to me very well") item reads: "I drank too much alcohol or I got drunk". Cronbach's alphas cannot be calculated for single-item scales, but one-year rank-order stability coefficients ranging from 0.65 to 0.77 indicated that this item was reliable. The raw scores on this single item were used in our analyses.

\section{Soft drug use}

Soft drug use in the last 6 months was measured with a single item adapted from the DBS [41]. This item is: "I used soft drugs (joints, marihuana, hashish)", and was scored on a 5-point Likert scale (ranging from "does not apply to me at all" to "applies to me very well"). One-year rank-order stability coefficients ranging from 0.71 to 0.86 indicated that this item was reliable. The raw scores on this single item were used in our analyses.

\section{Results}

To test spectrum, pathoplasty, and scar models, we ran six cross-lagged panel models in Mplus 7 [42]. Because Big Five traits and changes therein are well known to be associated with one another [43], one model included all Big Five domains. As externalizing behaviors are also associated with one another, all three externalizing behaviors were also included simultaneously in this model 


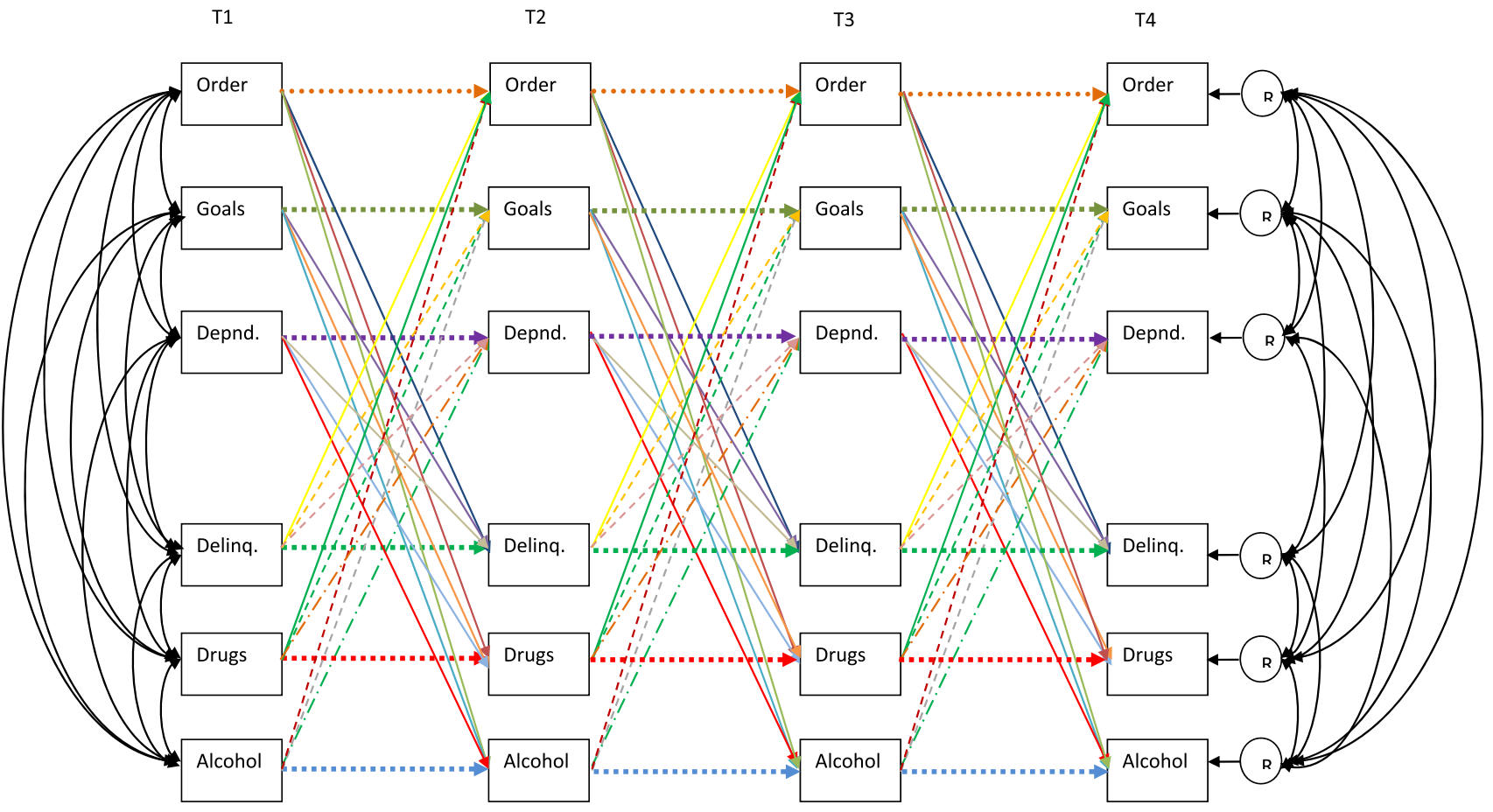

Fig. 1 Sample cross-lagged model with stability paths, initial correlations, correlated change, cross paths from personality (i.e., the conscientiousness facets) to externalizing behavior and vice versa. Order orderliness, Goals goal striving, Depnd. dependability, Delinq. delinquency, Drugs soft drugs use, Alcohol alcohol abuse, $R$ residual. Note that residuals and correlations among them (i.e., correlated change) were also examined at $\mathrm{T} 2$ and $\mathrm{T} 3$, but these residuals and correlations are not displayed in the figure to keep it readable. Correlated change coefficients were constrained to be equal across time, and so were stability paths and cross paths. Time-invariance constraints to the latter two types of paths are signified in the figure,

[44]. Five additional models were run for the facets. These models included all facets of one trait domain and the three externalizing behaviors. A sample cross-lagged panel model (i.e., the model for the conscientiousness facets) is depicted in Fig. 1.

To make these models as parsimonious as possible, we added several constraints. That is, correlated relative change coefficients (i.e., T2, T3, and T4 correlations; [e.g., 12]), stability paths (i.e., autoregressive paths) and cross paths from personality to adjustment and cross paths from adjustment to personality were constrained to be equal across measurement waves (i.e., time-invariant). A series of model comparisons was run to examine whether adding each of these constraints was justified. Thus, we pursued a hierarchical approach by adding time-invariance constraints to (a) correlated change coefficients, (b) stability paths, and (c) cross paths. Three criteria to compare nested models [45] were used: a significant Chi-square difference test [46], a difference in Comparative Fit Index (CFI) of $>0.010$ [47], and a difference in the Root Mean Square because paths that were constrained to be equal are equal in terms of color, pattern, and width. The Big Five trait domains and facets of the other domains were linked to externalizing behavior in similar models. That is, all possible initial correlations and correlated change associations were included, but we only considered cross paths from personality to externalizing behavior and vice versa. Thus, cross paths between externalizing behaviors (e.g., from soft drugs use to alcohol abuse and vice versa) and cross paths between personality trait domains or facets (e.g., from orderliness to goal striving and vice versa) were not included in the model, because these cross paths were not of interest in light of the research questions of this paper

Error of Approximation (RMSEA) of $>0.015$ [48]. Only if two of these criteria were satisfied, the less parsimonious model was favored over the more parsimonious model with constraints.

In a series of model comparisons, $\Delta$ CFIs were consistently $\leq 0.010$ and $\triangle$ RMSEAs were consistently $\leq 0.015$. Thus, at least two out of three fit criteria indicated that adding the previously described constraints to the correlated change coefficients did not result in a deteriorated fit for any of the models. We added constraints to the stability paths to the models in which the correlated change coefficients were constrained. Adding these additional constraints again did not lead to a significant deterioration in model fit for any of the models. In the resulting models, we constrained cross paths to be equal across time. As adding these constraints also did not lead to a significant deterioration of model fit, our final models contained timeinvariance constraints on correlated change coefficients, stability paths, and cross paths. It should be noted that constraining these associations to be time-invariant implies 
that unstandardized coefficients are set equal. Because standard errors tend to differ across the various time lags, standardized coefficients are typically still slightly different across time.

Fit of the final models was judged by assessing the Chi square, the CFI, and the RMSEA. Chi squares should be as small as possible, CFIs should be larger than 0.90, and RMSEAs should be smaller than 0.08 [49]. Fit statistics of all final models were adequate (see Table 2). In the following sections, we discuss associations in line with the

Table 2 Model fit statistics for cross-lagged panel models relating personality to adjustment $(N=485)$

\begin{tabular}{llllll}
\hline Model & $\chi^{2}$ & $d f$ & CFI & RMSEA & $\begin{array}{l}\text { 90 \% CI } \\
\text { RMSEA }\end{array}$ \\
\hline Big Five domains & $662.917 * * *$ & 386 & 0.966 & 0.038 & $0.033-0.043$ \\
Neuroticism facets & $527.561 * * *$ & 210 & 0.953 & 0.056 & $0.050-0.062$ \\
$\begin{array}{l}\text { Extraversion facets } \\
\text { Openness facets }\end{array}$ & $370.013 * * *$ & 210 & 0.958 & 0.051 & $0.044-0.057$ \\
$\begin{array}{l}\text { Agreeableness } \\
\text { facets }\end{array}$ & $290.331 * * *$ & 143 & 0.966 & 0.046 & $0.038-0.054$ \\
$\begin{array}{l}\text { Conscientiousness } \\
\text { facets }\end{array}$ & $473.546 * * *$ & 210 & 0.960 & 0.051 & $0.045-0.057$ \\
\hline$* p<0.05, * * p<0.001, * * * p<0.001$ & & & & \\
\hline
\end{tabular}

spectrum model, the pathoplasty model, and the scar model, respectively.

\section{Spectrum model}

Correlated change coefficients, as well as initial correlations, appear in Table 3. It should be noted that initial correlations are not particularly informative with respect to any of the theoretical models tested in this paper. However, for the spectrum model, correlated change is important [13]. As explained in the introduction, it is important to realize that when we refer to "change", we mean relative change (i.e., changes in the rank-order of individuals on a variable).

Changes in delinquency were positively associated with changes in neuroticism and all of its facets. In addition, we found negative correlated change of delinquency with agreeableness and its facet non-antagonistic orientation, and with conscientiousness and its facets orderliness and dependability. Changes in extraversion, openness, and their facets were not associated with changes in delinquency.

No evidence of correlated change of soft drugs use with neuroticism, openness, agreeableness, and their facets was found. There was positive correlated change between soft

Table 3 Initial correlations and correlated change in cross-lagged models of personality and adjustment $(N=485)$

\begin{tabular}{|c|c|c|c|c|c|c|c|c|c|c|c|c|}
\hline \multirow{2}{*}{$\begin{array}{l}\text { Persona } \\
\text { lity }\end{array}$} & \multicolumn{4}{|c|}{ Delinquency } & \multicolumn{4}{|c|}{ Soft drugs use } & \multicolumn{4}{|c|}{ Alcohol abuse } \\
\hline & $\mathrm{T} 1$ & $\mathrm{~T} 1-\mathrm{T} 2$ & $\mathrm{~T} 2-\mathrm{T} 3$ & T3-T4 & $\mathrm{T} 1$ & $\mathrm{~T} 1-\mathrm{T} 2$ & $\mathrm{~T} 2-\mathrm{T} 3$ & $\mathrm{~T} 3-\mathrm{T} 4$ & $\mathrm{~T} 1$ & $\mathrm{~T} 1-\mathrm{T} 2$ & $\mathrm{~T} 2-\mathrm{T} 3$ & T3-T4 \\
\hline $\mathrm{N}$ & $0.14 * *$ & $0.09 * * *$ & $0.12 * * *$ & $0.14 * * *$ & 0.02 & 0.00 & 0.00 & 0.00 & 0.02 & 0.02 & 0.02 & 0.03 \\
\hline $\mathrm{E}$ & $-0.13 * *$ & -0.01 & -0.01 & -0.01 & -0.04 & 0.02 & 0.02 & 0.03 & 0.06 & $0.08 * *$ & $0.09 * *$ & $0.10 * *$ \\
\hline $\mathrm{O}$ & $0.09 *$ & -0.01 & -0.01 & -0.02 & $0.12 * *$ & 0.03 & 0.04 & 0.05 & 0.05 & 0.04 & 0.04 & 0.05 \\
\hline A & $-0.35 * * *$ & $-0.10 * * *$ & $-0.14 * * *$ & $-0.16^{* * *}$ & $-0.26^{* * *}$ & -0.02 & -0.02 & -0.03 & $-0.35 * * *$ & -0.01 & -0.01 & -0.01 \\
\hline $\mathrm{C}$ & $-0.30 * * *$ & $-0.10^{* * *}$ & $-0.13 * * *$ & $-0.13 * * *$ & $-0.24 * * *$ & $-0.06^{*}$ & $-0.07 *$ & $-0.08 *$ & $-0.28 * * *$ & $-0.09 * * *$ & $-0.11 * * *$ & $-0.12 * * *$ \\
\hline Nsr & $0.16^{* * *}$ & $0.06^{*}$ & $0.08^{*}$ & $0.08^{*}$ & -0.01 & -0.01 & -0.02 & -0.02 & 0.05 & 0.00 & 0.00 & 0.00 \\
\hline $\mathrm{Na}$ & 0.04 & $0.08^{* * *}$ & $0.11 * * *$ & $0.11 * * *$ & -0.07 & 0.02 & 0.02 & 0.03 & -0.07 & 0.00 & 0.00 & 0.00 \\
\hline $\mathrm{Nd}$ & $0.16^{* * *}$ & $0.09^{* * *}$ & $0.11 * * *$ & $0.11 * * *$ & 0.05 & 0.02 & 0.02 & 0.02 & 0.05 & 0.03 & 0.03 & 0.03 \\
\hline Epa & $-0.18 * * *$ & -0.02 & -0.03 & -0.03 & -0.06 & 0.00 & 0.00 & 0.00 & -0.02 & 0.04 & 0.04 & 0.04 \\
\hline Eso & $-0.11^{*}$ & 0.00 & 0.00 & 0.01 & -0.04 & -0.01 & -0.01 & -0.01 & 0.05 & $0.06^{*}$ & $0.07 *$ & $0.08 *$ \\
\hline Eac & -0.07 & 0.01 & 0.01 & 0.01 & 0.03 & $0.05^{*}$ & $0.06^{*}$ & $0.08^{*}$ & $0.10^{*}$ & $0.09 * * *$ & $0.11 * * *$ & $0.12 * * *$ \\
\hline Oai & -0.03 & 0.04 & 0.05 & 0.05 & -0.03 & 0.03 & 0.03 & 0.04 & -0.04 & 0.01 & 0.01 & 0.01 \\
\hline Oii & 0.05 & -0.01 & -0.01 & -0.01 & 0.08 & 0.01 & 0.01 & 0.01 & 0.02 & 0.01 & 0.01 & 0.01 \\
\hline Oun & $0.14 * *$ & -0.02 & -0.03 & -0.03 & $0.14 * *$ & 0.04 & 0.04 & 0.06 & 0.06 & 0.04 & 0.05 & 0.06 \\
\hline Ano & $-0.36 * * *$ & $-0.13 * * *$ & $-0.18 * * *$ & $-0.18 * * *$ & $-0.23 * * *$ & -0.03 & -0.04 & -0.05 & $-0.20 * * *$ & 0.00 & 0.00 & 0.00 \\
\hline Apo & $-0.22 * * *$ & -0.01 & -0.01 & -0.01 & -0.08 & -0.01 & -0.02 & -0.02 & $-0.09^{*}$ & 0.00 & 0.00 & 0.00 \\
\hline Cor & $-0.29 * * *$ & $-0.10 * * *$ & $-0.14 * * *$ & $-0.14 * * *$ & $-0.18^{* * *}$ & $-0.06^{* *}$ & $-0.08 * *$ & $-0.09 * *$ & $-0.22 * * *$ & $-0.11 * * *$ & $-0.13 * * *$ & $-0.15 * * *$ \\
\hline $\mathrm{Cgs}$ & $-0.22 * * *$ & 0.00 & 0.00 & 0.00 & $-0.13 * *$ & -0.03 & -0.04 & -0.05 & $-0.15 * * *$ & $-0.06^{*}$ & $-0.06^{*}$ & $-0.07^{*}$ \\
\hline Cdp & $-0.24 * * *$ & $-0.08 * * *$ & $-0.11 * * *$ & $-0.12 * * *$ & $-0.17 * * *$ & -0.02 & -0.03 & -0.03 & $-0.20 * * *$ & $-0.05^{*}$ & $-0.06^{*}$ & $-0.07 *$ \\
\hline
\end{tabular}

Note. $N$ neuroticism, $N s r$ neuroticism - self-reproach, $N a$ neuroticism - anxiety, $N d$ neuroticism - depression, $E$ extraversion, Epa extraversion - positive affect, Eso extraversion - sociability, Eac extraversion - activity, $O$ openness, Oai openness - esthetic interests, Oii openness - intellectual interests, Oun openness - unconventionality, A agreeableness, Ano agreeableness - non-antagonistic orientation, Apo agreeableness - pro-social orientation, $C$ conscientiousness, Cor conscientiousness - orderliness, $C g s$ conscientiousness - goal striving, $C d p$ conscientiousness - dependability

$* p<0.05, * * p<0.001, * * * p<0.001$ 
Table 4 Cross-lagged effects of personality on adjustment $(N=485)$

\begin{tabular}{|c|c|c|c|c|c|c|c|c|c|}
\hline \multirow[t]{2}{*}{ Personality } & \multicolumn{3}{|c|}{ Delinquency } & \multicolumn{3}{|c|}{ Soft drugs use } & \multicolumn{3}{|c|}{ Alcohol abuse } \\
\hline & $\mathrm{T} 1-\mathrm{T} 2$ & $\mathrm{~T} 2-\mathrm{T} 3$ & $\mathrm{~T} 3-\mathrm{T} 4$ & $\mathrm{~T} 1-\mathrm{T} 2$ & $\mathrm{~T} 2-\mathrm{T} 3$ & T3-T4 & $\mathrm{T} 1-\mathrm{T} 2$ & $\mathrm{~T} 2-\mathrm{T} 3$ & T3-T4 \\
\hline Neuroticism & 0.01 & 0.01 & 0.01 & 0.00 & 0.00 & 0.00 & -0.01 & -0.01 & -0.01 \\
\hline Extraversion & -0.01 & -0.01 & -0.01 & -0.02 & -0.02 & -0.02 & 0.04 & 0.04 & 0.04 \\
\hline Openness & $0.06^{* *}$ & $0.06^{* *}$ & $0.07 * *$ & $0.05 *$ & $0.05^{*}$ & $0.05 *$ & 0.00 & 0.00 & 0.00 \\
\hline Agreeableness & -0.03 & -0.03 & -0.03 & 0.01 & 0.01 & 0.01 & -0.05 & -0.04 & -0.04 \\
\hline Conscientiousness & -0.03 & -0.03 & -0.03 & $-0.05 *$ & $-0.05^{*}$ & $-0.05^{*}$ & -0.04 & -0.04 & -0.04 \\
\hline $\mathrm{N}$ self-reproach & -0.01 & -0.01 & -0.01 & -0.03 & -0.03 & -0.03 & -0.01 & -0.01 & -0.01 \\
\hline $\mathrm{N}$ anxiety & 0.04 & 0.04 & 0.04 & 0.01 & 0.01 & 0.01 & 0.00 & 0.00 & 0.00 \\
\hline $\mathrm{N}$ depression & 0.01 & 0.01 & 0.01 & 0.05 & 0.04 & 0.05 & 0.02 & 0.01 & 0.01 \\
\hline E positive affect & $-0.07 *$ & $-0.06^{*}$ & $-0.06^{*}$ & -0.03 & -0.02 & -0.03 & -0.03 & -0.03 & -0.02 \\
\hline E sociability & 0.04 & 0.04 & 0.04 & -0.04 & -0.04 & -0.04 & -0.01 & -0.01 & 0.00 \\
\hline E activity & -0.01 & -0.01 & -0.01 & 0.02 & 0.02 & 0.02 & 0.05 & 0.04 & 0.04 \\
\hline $\mathrm{O}$ esthetic interests & 0.00 & 0.00 & 0.00 & 0.01 & 0.01 & 0.01 & -0.02 & -0.01 & -0.01 \\
\hline O intellectual interests & 0.04 & 0.04 & 0.05 & 0.01 & 0.01 & 0.01 & 0.03 & 0.02 & 0.03 \\
\hline O unconventionality & 0.03 & 0.03 & 0.03 & $0.06^{* *}$ & $0.05^{* *}$ & $0.05^{* *}$ & -0.01 & -0.01 & -0.01 \\
\hline A non-antagonistic orient. & $-0.06^{*}$ & $-0.06^{*}$ & $-0.06^{*}$ & -0.02 & -0.02 & -0.02 & -0.04 & -0.04 & -0.04 \\
\hline A pro-social orientation & 0.01 & 0.01 & 0.01 & 0.01 & 0.01 & 0.01 & -0.02 & -0.01 & -0.01 \\
\hline $\mathrm{C}$ orderliness & $-0.07 *$ & $-0.06^{*}$ & $-0.07 *$ & -0.05 & -0.04 & -0.04 & -0.02 & -0.02 & -0.02 \\
\hline $\mathrm{C}$ goal striving & 0.04 & 0.04 & 0.04 & -0.01 & -0.01 & -0.01 & -0.01 & -0.01 & -0.01 \\
\hline $\mathrm{C}$ dependability & -0.03 & -0.03 & -0.03 & -0.01 & -0.01 & -0.01 & -0.02 & -0.02 & -0.02 \\
\hline
\end{tabular}

$* p<0.05, * * p<0.001, * * * p<0.001$

drug use and the extraversion facet of activity. Finally, changes in conscientiousness and its facet orderliness were negatively associated with changes in soft drugs use.

Changes in alcohol abuse were not significantly associated with neuroticism, openness, agreeableness, or their facets. There was evidence for positive correlated change of alcohol abuse with extraversion and its facets sociability and activity. Changes in conscientiousness and its facets were negatively associated with changes in alcohol abuse.

Pathoplasty model

Cross-lagged effects of personality on externalizing behaviors support the pathoplasty model. As explained in the introduction, such effects would in our study indicate that the rankorder of a certain personality trait or facet (e.g., extraversion) on one measurement occasion is predictive of changes in the rank ordering of individuals on a certain type of externalizing behavior (e.g., delinquency) on the subsequent measurement occasion. These effects appear in Table 4.

The extraversion facet of positive affect negatively predicted delinquency, whereas openness at the domain level positively predicted delinquency. Delinquency was negatively predicted by the agreeableness facet nonantagonistic orientation and the conscientiousness facet orderliness.
Openness and its facet unconventionality positively predicted soft drug use. In addition, conscientiousness at the domain level negatively predicted soft drugs use. Table 4 shows that there were no significant predictors of alcohol use.

Scar model

Cross-lagged paths indicating effects of externalizing behaviors on personality support the scar model. In our study, such effects would indicate that the rank order of individuals on a certain type of externalizing behavior (e.g., delinquency) on one measurement occasion is predictive of changes in the rank ordering of individuals on a certain personality trait or facet (e.g., extraversion) on the subsequent measurement occasion. These effects are shown in Table 5.

Delinquency positively predicted neuroticism and all of its facets. Extraversion and its facet sociability, agreeableness and its facet non-antagonistic orientation, and conscientiousness and its facets orderliness and dependability were negatively predicted by delinquency.

Soft drugs use negatively predicted extraversion and its facet activity. Openness and its facets esthetic interests and intellectual interests were positively predicted by soft drug use. 
Table 5 Cross-lagged effects of adjustment on personality $(N=485)$

\begin{tabular}{|c|c|c|c|c|c|c|c|c|c|}
\hline \multirow[t]{2}{*}{ Personality } & \multicolumn{3}{|c|}{ Delinquency } & \multicolumn{3}{|c|}{ Soft drugs use } & \multicolumn{3}{|c|}{ Alcohol abuse } \\
\hline & $\mathrm{T} 1-\mathrm{T} 2$ & $\mathrm{~T} 2-\mathrm{T} 3$ & $\mathrm{~T} 3-\mathrm{T} 4$ & $\mathrm{~T} 1-\mathrm{T} 2$ & $\mathrm{~T} 2-\mathrm{T} 3$ & $\mathrm{~T} 3-\mathrm{T} 4$ & $\mathrm{~T} 1-\mathrm{T} 2$ & $\mathrm{~T} 2-\mathrm{T} 3$ & $\mathrm{~T} 3-\mathrm{T} 4$ \\
\hline Neuroticism & $0.08 * *$ & $0.06 * *$ & $0.06 * *$ & -0.01 & -0.01 & -0.01 & 0.01 & 0.01 & 0.01 \\
\hline Extraversion & $-0.06^{*}$ & $-0.05^{*}$ & $-0.05 *$ & $-0.05^{*}$ & $-0.04 *$ & $-0.05 *$ & 0.02 & 0.01 & 0.02 \\
\hline Openness & 0.03 & 0.02 & 0.02 & $0.04 *$ & $0.04 *$ & $0.04 *$ & 0.04 & 0.04 & 0.04 \\
\hline Agreeableness & $-0.08 * *$ & $-0.06^{* *}$ & $-0.06 * *$ & -0.01 & -0.01 & -0.01 & $-0.07 * *$ & $-0.06 * *$ & $-0.06^{* *}$ \\
\hline Conscientiousness & $-0.08 * *$ & $-0.06^{* *}$ & $-0.06 * *$ & -0.02 & -0.02 & -0.02 & $-0.07 * *$ & $-0.07 * *$ & $-0.06^{* *}$ \\
\hline $\mathrm{N}$ self-reproach & $0.08 * *$ & $0.07 * *$ & $0.06 * *$ & -0.02 & -0.02 & -0.02 & -0.02 & -0.02 & -0.02 \\
\hline $\mathrm{N}$ anxiety & $0.07 *$ & $0.06^{*}$ & $0.05 *$ & 0.00 & 0.00 & 0.00 & 0.00 & 0.00 & 0.00 \\
\hline $\mathrm{N}$ depression & $0.10 * * *$ & $0.08 * * *$ & $0.07 * * *$ & -0.01 & -0.01 & -0.01 & -0.03 & -0.03 & -0.03 \\
\hline E positive affect & -0.04 & -0.03 & -0.02 & 0.00 & 0.00 & 0.00 & -0.01 & -0.01 & 0.00 \\
\hline E sociability & $-0.07 * *$ & $-0.06^{* *}$ & $-0.05 * *$ & -0.03 & -0.03 & -0.03 & 0.03 & 0.03 & 0.03 \\
\hline E activity & -0.04 & -0.03 & -0.03 & $-0.05^{*}$ & $-0.05^{*}$ & $-0.05^{*}$ & $0.09 * * *$ & $0.08 * * *$ & $0.08 * * *$ \\
\hline $\mathrm{O}$ esthetic interests & 0.04 & 0.03 & 0.03 & $0.05^{*}$ & $0.04 *$ & $0.04 *$ & 0.00 & 0.00 & 0.00 \\
\hline $\mathrm{O}$ intellectual interests & 0.03 & 0.02 & 0.02 & $0.06 * *$ & $0.06 * *$ & $0.05 * *$ & -0.02 & -0.02 & -0.01 \\
\hline O unconventionality & 0.04 & 0.03 & 0.03 & 0.01 & 0.01 & 0.01 & $0.05^{*}$ & $0.05^{*}$ & $0.05^{*}$ \\
\hline A non-antagonistic orient. & $-0.09 * *$ & $-0.07 * *$ & $-0.06 * *$ & 0.01 & 0.01 & 0.01 & -0.04 & -0.04 & -0.04 \\
\hline A pro-social orientation & -0.02 & -0.02 & -0.02 & -0.01 & -0.01 & -0.01 & -0.04 & -0.04 & -0.04 \\
\hline $\mathrm{C}$ orderliness & $-0.11 * * *$ & $-0.08 * * *$ & $-0.07 * * *$ & -0.01 & 0.00 & 0.00 & $-0.05^{*}$ & $-0.04 *$ & $-0.04 *$ \\
\hline $\mathrm{C}$ goal striving & -0.01 & -0.01 & -0.01 & -0.01 & -0.01 & -0.01 & -0.04 & -0.04 & -0.04 \\
\hline $\mathrm{C}$ dependability & $-0.09 * *$ & $-0.07 * *$ & $-0.07 * *$ & -0.01 & -0.01 & -0.01 & -0.04 & -0.03 & -0.03 \\
\hline
\end{tabular}

$* p<0.05, * * p<0.001, * * * p<0.001$

Alcohol abuse positively predicted the extraversion facet of activity and the openness facet unconventionality. Agreeableness at the domain level, as well as conscientiousness and its facet orderliness were negatively predicted by alcohol abuse.

\section{Discussion}

The present study was the first to adopt a facet-level approach to personality in simultaneously examining spectrum, scar, and pathoplasty models regarding the linkages with externalizing behavior (i.e., minor delinquency, soft drugs use, and alcohol abuse). Overall, our results indicate that the fact that one personality facet underlying a particular trait domain is significantly associated with a specific externalizing behavior does not mean that all other facets underlying that trait domain are also significantly associated with that specific type of externalizing behavior. Thereby, we demonstrated that considering personality facets may be crucial to obtain a full understanding of these associations. The directionality of effects was complex, as longitudinal linkages between personality and externalizing behavior were best described by different models depending on the specific personality trait domain or facet and externalizing behavior that were being assessed. Below, our results are discussed in more detail.

Delinquency and personality

We found little evidence in favor of a pathoplasty model for describing the linkages between personality and delinquency. This seems to be in contrast to previous studies [14-16], but it should be noted that these studies only examined a pathoplasty model, and did not consider spectrum and scar models. There was still some evidence for pathoplasty effects that were not accompanied by scar or spectrum effects in our study, as individuals with high levels of openness at the domain level and low positive affect (a facet of extraversion) displayed relative increases in delinquency. The effect of low positive affect was in line with a previous study [16] that found low extraversion to be a risk factor for delinquency. Our study elaborated on this finding by suggesting that the positive affect facet may be the most important aspect of extraversion in this regard.

There was more evidence for the spectrum model and the scar model. Spectrum model explanations hold that delinquency may be a manifestation of extreme scores on particular personality traits [e.g., 10]. This should be evidenced by correlated change between delinquency and those particular traits [e.g., 13]. Our results suggest that 
delinquency may be a manifestation of high scores of neuroticism and its facets, low scores of agreeableness and its facet non-antagonistic orientation, and low scores of conscientiousness and its facets of orderliness and dependability. At the trait level, our findings are identical to those obtained in a meta-analysis [5]. However, they complement these findings by indicating which exact facets within these trait domains seem most important.

For neuroticism and all of its facets, extraversion and its facet sociability, agreeableness and its facet non-antagonistic orientation, and conscientiousness and its facets orderliness and dependability, we also found scar effects suggesting that those who exhibited relatively higher levels of delinquency were likely to become relatively more neurotic and less extraverted, agreeable, and conscientious. A previous study [17] also found such scar effects for domains related to conscientiousness (i.e., Constraint) and neuroticism (i.e., Negative Emotionality), but found little evidence for scar effects on a domain related to extraversion (i.e., Positive Emotionality). Scar effects had not yet been tested for agreeableness, but our findings suggest that this personality domain may also be important to consider.

Our findings suggest that an antagonistic orientation and a lack of orderliness may be particularly detrimental, as we found evidence for both pathoplasty and scar effects for these facets. Specifically, antagonistic and disorderly individuals were more likely to exhibit relative increases in delinquency, which made them more likely to exhibit further relative decreases in orderliness and increases in antagonism, which, in turn, made them yet more likely to display further relative increases in delinquency. The finding for orderliness seems to converge with studies that found that living in a chaotic household is a risk factor for externalizing problems [e.g., 50]. A non-antagonistic orientation as operationalized in facet-level approaches to the NEO-FFI [27, 35] entails assets that are negatively associated with "dark" personality traits such as Machiavellianism and psychopathy [e.g., 51]. Collectively, these findings therefore suggest that both a lack of structure in life and the presence of "dark" traits may put individuals at risk of ending up in a negative spiral amounting to delinquency.

\section{Soft drugs use and personality}

For soft drugs use, we found little evidence for a spectrum model. In line with previous studies [3, 18, 19], soft drugs use appears to be a manifestation of higher scores on the extraversion facet of activity and lower scores on conscientiousness. For conscientiousness, previous studies pointed to the importance of facets related to dependability (i.e., dutifulness and responsibility; [3, 18]). In contrast to previous studies, we found that orderliness, and not dependability, was strongly associated with soft drugs use. This may be due to the fact that dependability was unreliably measured at some measurement waves in the present study.

There was also relatively little evidence for a pathoplasty model, as only individuals who scored high on openness and its facet unconventionality, and low on conscientiousness were somewhat more likely to become involved in soft drugs use. conscientiousness [20] and the openness facet unconventionality [31] had also been identified as risk factors for soft drugs use in previous studies. The association with unconventionality in the present study should still be interpreted cautiously, as this facet scale was measured unreliably. Overall, the relatively little evidence we found for spectrum and pathoplasty models suggests that being or becoming involved in soft drugs use in late adolescence and young adulthood is rather independent of one's personality, at least as measured in the present study.

From our results, it appears that the longitudinal associations between soft drugs use and personality are best described by a scar model, in which soft drugs use predicts personality change. In this case, such "scar" effects were not necessarily "bad", as soft drugs use predicted increases in openness and its facets esthetic and intellectual interests. This suggests that soft drugs use might indeed be a kind of experimentation related to the identity exploration process $[2,52]$. The fact that direct measures of identity exploration have also been found to predict increases in openness to Experience [53] seems to support this interpretation. However, it should be noted that the magnitude of these effects is rather modest.

Soft drugs use also had some adverse scar effects, as it predicted relative decreases in extraversion and its facet activity, but again the magnitude of these effects was rather modest. The one previous study that examined a scar model [22] did not include extraversion or its facets, thus replication of this effect seems warranted before definite conclusions are drawn. Overall, however, it seems safe to conclude that soft drugs use might leave a mark on one's personality, but that this mark seems to be not exclusively negative.

Alcohol abuse and personality

Our most striking finding with regard to the linkages between personality and alcohol abuse concerns the fact that we found no significant pathoplasty effects. A previous study that simultaneously examined scar and pathoplasty models [22] also found little evidence for pathoplasty effects. This supports the view that there is no particular constellation of traits, or so-called "addictive personality", which leaves one vulnerable to alcohol abuse [54]. At least one previous study 
[25] found some evidence for a pathoplasty model, but that study focused on the onset of alcohol use, and not on the severity of alcohol abuse. Still, more studies are needed before pathoplasty model explanations of associations between personality and alcohol abuse are refuted.

We found some evidence for a spectrum model suggesting that alcohol abuse might be a behavioral manifestation of high scores on extraversion and its facets sociability and activity, and low scores on conscientiousness and all of its facets. Previous studies also found linkages of alcohol abuse with low conscientiousness $[4,18,23,24]$ and high extraversion $[20,21]$. Our results also converge with those of McAdams and Donnellan [32] by suggesting that facets related to sociability are associated with alcohol abuse. Sociability is often considered a positive asset, as sociable individuals tend to have more friends [e.g., 55]. However, alcohol abuse is strongly affected by peer influences in friendship networks [56], which might explain why a seemingly positive asset like sociability might be associated with becoming involved in alcohol abuse.

There was also some evidence for scar effects of alcohol abuse. Some of these effects seemed favorable, as alcohol abuse positively predicted the extraversion facet of activity and the openness facet of unconventionality. The association with unconventionality should be interpreted cautiously given its poor reliability, but overall these associations suggest that alcohol abuse, much like soft drugs use, may sometimes be perceived as mere experimenting, and thus as part of the identity exploration process [52]. However, more so than with soft drugs use, adverse scar effects of alcohol abuse seem to outweigh potential benefits. Specifically, alcohol abuse seems to predict changes towards a relatively less agreeable and less conscientious personality. These findings are in line with previous studies $[22,25]$. Still, more studies are needed to uncover all potentially adverse effects of alcohol abuse on personality development. Such research seems particularly warranted because of the importance of a healthy personality in young adult adjustment and dealing with the challenges of this period in the lifespan [e.g., 53, 57].

\section{Clinical implications}

Results of the present study also point to several potential clinical implications. First, the fact that some facets underlying specific trait domains were significantly associated with externalizing behaviors whereas other facets underlying the same trait domain were not, suggests it may be more efficient to target specific facets (e.g., orderliness) instead of broad trait domains (e.g., conscientiousness) in preventive interventions. Before such a more refined approach can be implemented, more studies are needed to ascertain that specific facets are replicable risk factors. Such efforts are likely to be worthwhile, as facets are associated with more specific behaviors and experiences than broad trait domains are. Therefore, reliable facet-level information may make it easier to choose specific interventions [26].

Second, our study and several previous studies [e.g., 22] suggest that there is little evidence for risk factors in the personality realm for alcohol abuse. Thus, when treating young adults with drinking problems, there may be limited utility in looking for personality-related risk factors. Instead, the several scar effects we found suggest that alcohol abuse itself should be treated directly to avoid damaging effects on a client's personality.

Finally, the fact that we found some evidence for transactional effects with personality traits and externalizing behaviors mutually affecting one another, suggests that individuals can sometimes enter a negative spiral. This was, for example, the case with regard to the associations of orderliness with delinquency. Especially when there is evidence for such transactional effects, it is of particular importance to break this negative spiral as soon as possible and stop externalizing behaviors from exacerbating.

\section{Limitations}

Several limitations need to be recognized. First, we relied on a Belgian college student sample predominantly consisting of females. It is unclear to what extent our results are replicable in more representative samples and samples from other countries. However, it should be noted that previous studies found few gender differences in associations between personality and adjustment [e.g., 12]. In addition, many of our findings at the domain level were in line with previous studies, suggesting that our facet-level findings may also be generalizable.

A second, related, issue is that we only focused on a non-clinical sample. For that reason, we refer to (minor) delinquency, soft drugs use, and alcohol abuse as externalizing behavior instead of disorders throughout the present study. Future studies will need to sort out whether the same mechanisms that we found also apply to clinical populations.

A third limitation is that our findings do not provide evidence for pathoplasty effects or scar effects in the most literal sense. For example, it cannot be concluded from our findings that individuals will be likely to exhibit an absolute increase in delinquency when they exhibit high levels of openness. Instead, the cross-lagged models we used are only suitable for predicting relative changes in variables (i.e., delinquency) [36]. This implies that a positive path from openness to delinquency does not indicate that individuals exhibiting high levels of openness are likely to exhibit absolute increases in delinquency. Such a finding could just as well indicate that individuals with high levels 
of openness tend to exhibit smaller decreases in delinquency. Models focusing on latent change (e.g., [13]) come with similar problems. In such models, a positive effect of openness on delinquency could also indicate that individuals with higher levels of openness exhibit smaller decreases in delinquency. One way in which one would be certain to predict actual increases would be to categorize individuals into groups of increasers, non-changers, and decreasers, and predict group membership. Methods to make such groups in a reliable manner are available (e.g., reliable change index; [58]), but have the disadvantage that much information regarding individual differences in the amount of change is lost. Therefore, we think that crosslagged panel models and models focusing on latent changes are still the best available methods for examining pathoplasty and scar models.

Fourth, we solely relied on self-reported data, which make our results susceptible to shared-method effects. By controlling for initial correlations and correlated change, cross-lagged models largely partial out such effects on cross-lagged paths [e.g., 36]. However, like the authors of a previous study [13], we interpreted correlated change as evidence for the spectrum model. As noted in that study, it is hard to control for shared-method variance when examining the spectrum model. Future studies could address this issue by including other-reported data on externalizing behavior and personality, but bear in mind that such data are also slightly biased [59].

Fifth, soft drugs use and alcohol abuse were measured with single items. Although the use of such measures is relatively common when examining substance (ab)use [e.g., 21], using multiple-item scales still seems advisable because such instruments allow for checks of internal consistency. On the other hand, we still managed to demonstrate the reliability of our measures by showing that their test-retest correlations were high.

Sixth, the use of the NEO-FFI to measure personality at a facet level was a limitation. The best available measures to examine personality at a facet level are the related NEOPI-R and NEO-PI-D3 [26, 33, 34], which distinguish six personality facets per domain instead of the only two or three facets per domain that can be distinguished with the NEO-FFI. Thus, to optimally examine what facet of a trait domain is driving a particular association with externalizing behavior, the NEO-PI-R or NEO-PI-3 should have been used. In addition, these measures have been specifically developed to examine personality at the facet level, whereas the facets of the NEO-FFI have been constructed with post hoc factor analyses [27, 35]. This post hoc approach to constructing facet scales had two additional disadvantages. First, some of the facet scales only consisted of a few (i.e., three) items. Second, the items that represented the facet scales were sometimes quite diverse.
For example, the unconventionality scale included items on daydreaming and on whether participants think that decisions regarding moral issues should be left to religious leaders. Although most facet scales were more consistent in content, this diversity and the small number of items per scale has likely contributed to the low reliability of some of the facet scales in the present study and previous studies [27, 35]. Especially the Cronbach's alphas of the unconventionality facet and the pro-social behavior facet were problematic. Therefore, results involving these two facets should be interpreted cautiously.

Although the facets of the other NEO-FFI domains worked reasonably well, using this relatively brief measure was mainly a strength from a practical point-of-view. That is, a traditional facet-level measure like the NEO-PI-R has four times as many (i.e., 240) items and are therefore hard to include in longitudinal studies that also measure other constructs. To overcome the practical problems with lengthy measures and the validity and reliability problems with the NEO-FFI, new brief measures need to be developed that are directly intended to capture personality at a facet level.

\section{Conclusion}

Notwithstanding the aforementioned limitations, the present study contributed to the literature on personality and externalizing behaviors in several ways. First, we demonstrated the incremental value of personality facets over broad trait domains by showing that often some, but not all, facets underlying a specific trait domain were significantly associated with specific externalizing behaviors. Furthermore, we showed that facets can be associated with externalizing behavior, even if the overarching trait domain is not. Although there are some clear problems with unreliable facet scales (i.e., unconventionality and prosocial orientation), we did demonstrate that the facet scales of the relatively brief 60-item NEO-FFI generally worked quite well. Thus, despite the limitations of some scales, most of the facet scales of the NEO-FFI provide researchers who already have (longitudinal) data on this measure with the opportunity to (re-)examine their research questions at a facet level in a reliable and valid manner. Finally, our results point to the complexity of the linkages between personality and externalizing behavior. Depending on the personality trait domain or facet and the externalizing behavior that are assessed, different models (i.e., spectrum, pathoplasty, or scar models) may best describe these linkages.

Conflict of interest On behalf of all authors, the corresponding author states that there is no conflict of interest. 


\section{References}

1. Arnett JJ (2000) Emerging adulthood: a theory of development from the late teens through the twenties. Am Psychol 55:469-480

2. Arnett JJ (2005) The developmental context of substance use in emerging adulthood. J Drug Issues 35:235-253

3. Terraciano A, Lockenhoff CE, Crum RM, Bienvenu J, Costa PT, Jr (2008). Five-factor model personality profiles of drug users. BMC Psychiatry 8:22

4. Kotov R, Gamez W, Schmidt F, Watson D (2010) Linking "big" personality traits to anxiety, depressive, and substance use disorders: a meta-analysis. Psychol Bull 136:768-821

5. Miller JD, Lynam D (2001) Structural models of personality and their relation to antisocial behavior: a meta-analytic review. Criminology 39:765-798

6. Goldberg LR (1990) An alternative "description of personality": the Big-Five factor structure. J Pers Soc Psychol 59:1216-1229

7. McCrae RR, Costa PT (1987) Validation of the five-factor model of personality across instruments and observers. J Pers Soc Psychol 52:81-90

8. Caspi A, Roberts BW, Shiner RL (2005) Personality development: stability and change. Annu Rev Psychol 56:453-484

9. Krueger RF, Tackett JL (2003) Personality and psychopathology: working toward the bigger picture. J Pers Disord 17:109-128

10. Tackett JL (2006) Evaluating models of the personality-psychopathology relationship in children and adolescents. Clin Psychol Rev 26:584-599

11. Ormel J, Oldehinkel AJ, Vollebergh W (2004) Vulnerability before, during, and after a major depressive episode. Arch Gen Psychiatry 61:990-996

12. Klimstra TA, Akse J, Hale WW III, Raaijmakers QAW, Meeus WHJ (2010) Longitudinal associations between Big Five personality traits and problem behavior in adolescence. J Res Pers 44:273-284

13. De Bolle M, Beyers W, De Clercq B, De Fruyt F (2012) General personality and psychopathology in referred and nonreferred children and adolescents: an investigation of continuity, pathoplasty, and complication models. J Abnorm Psychol 121:958-970

14. Mottus R, Guljajev J, Allik J, Laidra K, Pullmann H (2012) Longitudinal associations of cognitive ability, personality traits and school grades with antisocial behaviour. Eur J Pers 26:56-62

15. Shiner RL (2000) Linking childhood personality with adaptation: evidence for mo continuity and change across time into late adolescence. J Pers Soc Psychol 78:310-325

16. Prinzie P, van der Sluis CM, de Haan AD, Dekovic M (2010) The mediational role of parenting on the longitudinal relation between child personality and externalizing behavior. J Pers 78:1301-1324

17. Ge X, Conger RD (1999) Adjustment problems and emerging personality characteristics from early to late adolescence. Am J Commun Psychol 27:429-459

18. Bogg T, Roberts BW (2004) Conscientiousness and health-related behaviors: a meta-analysis of the leading behavioral contributors to mortality. Psychol Bull 130:887-919

19. Fridberg DJ, Vollmer JM, O’Donnell BF, Skosnik PD (2011) Cannabis users differ from non-users on measures of personality and schizotypy. Psychiatry Res 186:46-52

20. Grekin ER, Sher KJ, Wood PK (2006) Personality and substance dependence symptoms: modeling substance-specific traits. Psychol Addict Beh 20:415-424

21. Hampson SE, Tildesley E, Andrews JA, Luyckx K, Mroczek DK (2010) The relation of change in hostility and sociability during childhood to substance use in mid adolescence. J Res Pers 44:103-114

22. Chassin L, Dmitrieva J, Modecki K, Steinberg L, Cauffman E, Piquero AR, Knight GP, Losoya SH (2010) Does adolescent alcohol and marijuana use predict suppressed growth in psychosocial maturity among male juvenile offenders? Psychol Addict Beh 24:48-60

23. Malouff JM, Thorsteinsson EB, Rooke SE, Schutte NS (2007) Alcohol involvement and the five-factor model of personality: a meta-analysis. J Drug Educ 37:277-294

24. Littlefield AK, Sher KJ, Wood PK (2010) A personality-based description of maturing out of alcohol problems: extension with a five-factor model and robustness to modeling challenges. Addict Beh 35:948-954

25. Hicks BM, Durbin CE, Blonigen DM, Iacono WG, McGue M (2011) Relationship between personality change and the onset and course of alcohol dependency in young adulthood. Addiction 107:540-548

26. Costa PT Jr, McCrae RR (1995) Domains and facets: hierarchical personality assessment using the revised NEO personality inventory. J Pers Assess 64:21-50

27. Saucier G (1998) Replicable item-cluster subcomponents in the NEO Five-Factor Inventory. J Pers Assess 7:263-276

28. Soto CJ, John OP (2009) Ten facet scales for the Big five inventory: convergence with NEO PI-R facets, self-peer agreement, and discriminant validity. J Res Pers 43:84-90

29. Tellegen A (1982) Brief manual for the Multidimensional Personality Questionnaire. Unpublished manuscript, University of Minnesota, Minneapolis

30. Krueger RF (1999) Personality traits in late adolescence predict mental disorders in early adulthood: a prospective-epidemiological study. J Pers 67:39-65

31. Gorman DM, Derzon JH (2002) Behavioral traits and marijuana use and abuse: a meta-analysis of longitudinal studies. Addict Beh 27:193-206

32. McAdams KK, Donnellan MB (2009) Facets of personality and drinking in first-year college students. Pers Individ Diff 46:207-212

33. McCrae RR, Costa PT Jr, Martin TA (2005) The NEO PI-3: a more readable revised NEO Personality Inventory. J Pers Assess $84: 261-270$

34. Costa PT Jr, McCrae RR (1992) Revised NEO Personality Inventory (NEO-PI-R) and the Five Factor Inventory (NEO-FFI): Professional manual. Psychological Assessment Resources, Odessa

35. Chapman BP (2007) Bandwidth and fidelity on the NEO-Five Factor Inventory: replicability and reliability of Saucier's (1998) ítem cluster subcomponents. J Pers Assess 88:220-234

36. Neyer FJ, Asendorpf JB (2001) Personality-relationship transaction in young adulthood. J Pers Soc Psychol 81:1190-1204

37. Luyckx K, Goossens L, Soenens B (2006) A developmentalcontextual perspective on identity construction in emerging adulthood: change dynamics in commitment formation and commitment evaluation. Dev Psychol 42:366-380

38. Little R (1988) A test of missing completely at random for multivariate data with missing values. J Am Stat Assoc 83: $1198-1202$

39. Bollen K (1989) Structural equations with latent variables. Wiley, New York

40. Hoekstra HA, Ormel J, De Fruyt F (1996) NEO persoonlijkheids vragenlijsten: NEO-PI-R, NEO-FFI. Handleiding [NEO personality questionnaires: NEO-PI-R and NEO-FFI-Manual]. Swets \& Zeitlinger, Lisse

41. Weinmann LL (1992) Patterns of change in middle adolescent adjustment: The role of relationships with parents and peers. Dissertation abstracts international, vol. 53, pp. 2565B-2566B (University Microfilms No. DA92-18128)

42. Muthén LK, Muthén BO (2012) Mplus user's guide, 7th edn. Muthén \& Muthén, Los Angeles 
43. Klimstra TA, Bleidorn W, Asendorpf JB, van Aken MAG, Denissen JJA (2013) Correlated change of big five personality traits across the lifespan: a search for determinants. J Res Pers 47:768-777

44. D'Amico EJ, Edelen MO, Miles JNV, Morral AR (2008) The longitudinal association between substance use and delinquency among high-risk youth. Drug Alcohol Depend 93:85-92

45. Vandenberg RJ, Lance CE (2000) A review and synthesis of the measurement invariance literature: suggestions, practices, and recommendations for organizational research. Organ Res Methods 3:4-70

46. Steiger JH, Shapiro A, Browne MW (1985) On the multivariate asymptotic distribution of sequential Chi square statistics. Psychometrika 50:253-264

47. Cheung GW, Rensvold RB (2002) Evaluating goodness-of-fit indexes for testing measurement invariance. Struct Equ Model 9:233-255

48. Chen FF (2007) Sensitivity of goodness of fit indexes to lack of measurement invariance. Struct Equ Model 14:464-504

49. Kline RB (2005) Principles and practice of structural equation modeling, 2nd edn. Guilford Press, New York

50. Wang Z, Deater-Deckard K, Petrill SA, Thompson LA (2012) Externalizing problems, attention regulation, and household chaos: a longitudinal behavioral genetic study. Dev Psychopathol 24:755-769

51. Paulhus DL, Williams KM (2002) The dark triad of personality: narcissism, machiavellianism, and psychopathy. J Res Pers 36:556-563
52. Erikson EH (1950) Childhood and society. Norton, New York

53. Luyckx K, Soenens B, Goossens L (2006) The personalityidentity interplay in emerging adult women: convergent findings from complementary analyses. Eur J Pers 20:195-215

54. Nathan P (1988) The addictive personality is the behavior of the addict. J Consult Clin Psychol 56:183-188

55. Puckett MB, Aikins JW, Cillessen AHN (2008) Moderators of the association between relational aggression and perceived popularity. Aggress Behav 34:563-576

56. Giletta M, Scholte RHJ, Prinstein MJ, Engels RCME, Rabaglietti E, Burk WJ (2012) Friendship context matters: examining the domain specificity of alcohol and depression socialization among adolescents. J Abnorm Child Psychol 40:1027-1043

57. Robins RW, Noftle EE, Trzesniewski KH, Roberts BW (2005) Do people know how their personality has changed? Correlates of perceived and actual personality change in young adulthood. J Pers 73:489-522

58. Jacobsen NS, Truax P (1991) Clinical significance: a statistical approach to defining meaningful change in psychotherapy research. J Consult Clin Psychol 59:12-19

59. Wood D, Harms P, Vazire S (2010) Perceiver effects as projective tests: what your perceptions of others say about you. J Pers Soc Psychol 99:174-190 